Pacific Journal of Mathematics

ON ELEMENTARY IDEALS OF POLYHEDRA IN THE 


\section{ON ELEMENTARY IDEALS OF POLYHEDRA IN THE 3-SPHERE}

\section{SHIN'ICHI KINOSHITA}

Let $K$ be a polygonal simple closed curve (a knot) in a 3 -sphere $S^{3}$. For each nonnegative integer $d$ the $d$ th elementary ideal $E_{d}$ of $K$ in the integral group-ring over an infinite cyclic group is defined by $R$. H. Fox. The ideal $E_{d}$ of $K$ is a topological invariant of the position of $K$ in $S^{3}$. This method has been applied to various more general settings, for instance, links in $S^{3}, S^{n-2}$ in $S^{n}(n>2)$ and etc. In this paper the $d$ th elementary ideals $E_{d}(l)$ are associated to each $(n-2)$-dimensional integral cycle $l$ on a polyhedron $L$ in an $n$-sphere $S^{n}(n>2)$ that does not separate $S^{n}$. The collection of $E_{d}(l)$ for all possible $l$ on $L$ forms a toplogical invariant of the position of $L$ in $S^{n}$.

In $\S 2$ we prove theorems of the $d$ th elementary ideal $E_{d}(l)$ associated with an $(n-2)$-dimensional integral $l$ on a polyhedron $L$ in $S^{n}$ that does not separate $S^{n}(n>2)$. In $\S 3$ we will consider to the case of polyhedra in $S^{3}$. After studying an example of a $\theta$-curve in $S^{3}$ in $\S 4$, we reconsider knots and links from this point of view in $\S 5$. In $\S 6$ we will give a remark on a Torres' formula for a link in $S^{3}$ ([7]) from this point of view.

Our discussion will be based on Fox's free differential calculus ([1], [2], [3]), though other methods, especially the covering space technique, would also be helpful. We need some minor adjustment of free differential calculus that will be given in $\S 1$.

The method of the paper is essentially different from that of [4], though a close relation between them will be observed.

1. From free differential calculus, Let $G$ be a group with a presentation $\left(x_{1}, \cdots, x_{m} ; r_{1}, \cdots, r_{n}\right)(n \leqq \infty)$. Let $H$ be a multiplicative abelian group and $\psi$ a homomorphism of $G$ into $H$. Let $\phi$ be the canonical homomorphism of the free group $F\left(x_{1}, \cdots, x_{m}\right)$ onto $G$. These homomorphism $\phi$ and $\psi$ are naturally extended to ring homomorphisms of the integral group-ring $J F$ onto $J G$ and of $J G$ into $J H$, respectively. Using Fox's free differential calculus ([1], [2], [3]), we have an $n \times$ $m$ matrix $A(G, \psi)=\left(r_{i j}\right)$, where

$$
r_{i j}=\left(\frac{\partial r_{i}}{\partial x_{j}}\right)^{\psi \phi \dot{\phi}} \in J H
$$

Generally, let $\left(r_{i j}\right)$ be an $n \times m$ matrix $(n \leqq \infty)$ over a commutative 
ring $R$ with unity. Then for each nonnegative integer $d$ the $d$ th elementary ideal $E_{d}$ of the matrix $\left(r_{i_{j}}\right)$ is the ideal of $R$ generated by all minors of order $m-d$, if $m-d>0$ and $n-(m-d) \geqq 0$. If $m-d>0$, but $n-(m-d)<0$, let $E_{d}=(0)$. If $m-d \leqq 0$, let $E_{d}=(1)$.

It was proved in [3] that the $d$ th elementary ideal $E_{d}$ of the matrix $A(G, \alpha)$ is an invariant of the group $G$, where $\alpha$ is the abelianizer of $G$. Since there is a unique homomorphism $\sigma$ of the abelianization of $G$ into $H$ with $\psi=\sigma \alpha$, the $d$ th elementary ideal $E_{d}(G, \psi)$ of the matrix $A(G, \psi)$ is an invariant of $G$ with respect to $\psi$.

Now let us consider the following diagram:

$$
G_{1} \stackrel{\varphi}{\longrightarrow} G_{2} \stackrel{\sigma}{\longrightarrow} H
$$

where $G_{1}$ and $G_{2}$ are groups, $H$ a multiplicative abelian group, and $\psi$ is a homomorphism of $G_{1}$ onto $G_{2}$ and $\sigma$ of $G_{2}$ into $H$. Then we have the following theorem.

\section{THEOREM 1. $E_{d}\left(G_{1}, \sigma \psi\right) \subset E_{d}\left(G_{2}, \sigma\right)$.}

Proof. Let $\left(x_{1}, \cdots, x_{m}: r_{1}, \cdots, r_{n}\right)(n \leqq \infty)$ be a presentation of $G_{1}$. Then $x_{1}^{\psi}, \cdots, x_{m}^{\psi}$ are generators of $G_{2}$, since $\psi$ is a homomorphism of $G_{1}$ onto $G_{2}$, and $r_{1}^{\psi}, \cdots, r_{n}^{\psi}$ are some of the relators of $G_{2}$. From this it follows that one of $A\left(G_{1}, \sigma \psi\right)$ is a submatrix of one of $A\left(G_{2}, \sigma\right)$, where the both matrices have the same number of columns. The theorem is now clear.

A trivializer of a group $G$ is a homomorphism of $G$ onto the trivial group that consists of only one element. Any trivializer will be denoted by the same notation $o$ in this paper. Further, the groupring $J o(G)$ will be identified with $J$.

For the convenience of the readers we refer to the following two theorems from free differential calculus ([2]).

TheOREM A (Fox). Let $G$ be a group and o a trivializer of $G$. Then a matrix $A(G, o)$ is elementarily equivalent (see [1], [3]) to the following matrix:

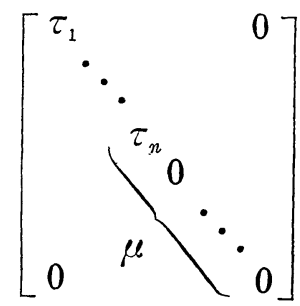

where $\tau_{1}, \cdots, \tau_{n}$ are torsion numbers and $\mu$ the Betti number of the 
abelianization of $G$. (We assume that the abelianization of $G$ is finitely generated.)

THEOREM B (Fox). Let $H$ be a multiplicative free abelian group of rank $\mu(\mu \geqq 1)$ with generators $t_{1}, \cdots, t_{\mu}$ and let $i$ be the identity of $H$. Then we have

$$
\left\{\begin{array}{l}
E_{0}(H, i)=(0), \\
E_{d}(H, i)=\left(t_{1}-1, \cdots, t_{\mu}-1\right)^{\mu-d}, \text { if } 1 \leqq d<\mu, \\
E_{d}(H, i)=(1), \text { if } d \geqq \mu .
\end{array}\right.
$$

2. On polyhedra in $S^{n}$. Let $L$ be a polyhedron in an $n$-sphere $S^{n}(n \geqq 3)$ that does not separate $S^{n}$, and let $G_{L}$ be the fundamental group of $S^{n}-L$. Let $l$ be an $(n-2)$-dimensional cycle with integral coefficients on $L$. There is a homomorphism $\psi$ of the group $G_{L}$ into the multiplicative infinite cyclic group $H$ generated by $t$ such that for each $g \in G_{L}$,

$$
g^{\psi}=t^{\operatorname{link}(g, l)},
$$

where link $(g, l)$ is the linking number between $g$ and $l$ in $S^{n}$. Since the $d$ th elementary ideal $E_{d}\left(G_{L}, \psi\right)$ is an invariant of the group $G_{L}$ with respect $\psi$, it is a topological invariant of $S^{n}-L$ with respect to $l$ on $L$, and from this it follows that $E_{d}\left(G_{L}, \psi\right)$ is a topological invariant of the position of $l$ on $L$ in $S^{n}$. We will denote it by $E_{d}(l)$. If two $(n-2)$-cycles $l$ and $l^{\prime}$ are homologous on $L$, then the corresponding $d$ th elementary ideals $E_{d}(l)$ and $E_{d}\left(l^{\prime}\right)$ are the same. The collection of $E_{d}(l)$ for all possible $(n-2)$-cycles $l$ on $L$ forms a topological invariant of the position of $L$ in $S^{n}$.

THEOREM 2. Let $L$ be a polyhedron in an $n$-sphere $S^{n}(n \geqq 3)$ that does not separate $S^{n}$. Let $p_{n-2}(L)$ be the $(n-2)$-dimensional Betti number of $L$ and $\tau_{1}, \tau_{2}, \cdots, \tau_{r}$ are $(n-3)$-dimensional torsion numbers with $\tau_{i} \mid \tau_{i+1}(i=1,2, \cdots, r-1)$. Then for each $(n-2)$-dimensional cycle $l$ on $L$ the dth elementary ideal $E_{d}(l)$ of $l$ on $L$ in $S^{n}$ satisfies the following conditions:

$$
\left\{\begin{aligned}
\left(E_{d}(l)\right)^{\circ}= & (0), \text { if } d<p_{n-2}(L), \\
\left(E_{d}(l)\right)^{\circ}= & \tau_{1} \tau_{2} \cdots \tau_{m}, \text { where } m=r-\left(d-p_{n-2}(L)\right), \\
& \text { if } p_{n-2}(L) \leqq d<p_{n-2}(L)+r, \\
\left(E_{d}(l)\right)^{\circ}= & (1), \text { if } d \geqq p_{n-2}(L)+r .
\end{aligned}\right.
$$

Proof. By the Alexander duality theorem we have

$$
p_{1}\left(S^{n}-L\right)=p_{n-2}(L)
$$


and

$$
T_{1}\left(S^{n}-L\right) \approx T_{n-3}(L),
$$

where $T_{i}(K)$ is the $i$-dimensional torsion group of a complex $K$. Since the abelianizations of $\pi\left(S^{n}-L\right)$ is the 1-dimensional homology group of $S^{n}-L$, the theorem is clear by Theorem A.

Especially if $l_{0}$ is the $(n-2)$-cycle on $L$ such that the coefficients of $l_{0}$ on every $(n-2)$-simplexes of $L$ are 0 , then for every $g \in G_{L}$ we have link $\left(g, l_{0}\right)=0$. Hence the homomorphism \& of $G_{L}$ into $H$ with respect to $l_{0}$ is a trivializer. Therefore, we have the following theorem.

Theorem 3. Let $L$ be a polyhedron in $S^{n}(n \geqq 3)$ that does not separate $S^{n}$ and $l_{0}$ the $(n-2)$-cycle on $L$ with coefficients 0 on every $(n-2)$-simplexes on $L$. Then we have

$$
E_{d}\left(l_{0}\right)=\left(E_{d}(l)\right)^{\circ},
$$

where $l$ is an $(n-2)$-cycle on $L$.

Now let $\mu$ be the number of components of $L$ (i.e. $\mu=p_{0}(L)$ ) and $L_{i}(i=1,2, \cdots, \mu)$ the $i$ th component of $L$. Then an $(n-2)$-cycle $l$ on $L$ can be expressed as $\sum_{i=1}^{\mu} l_{i}$, where $l_{i}(i=1,2, \cdots, \mu)$ is an $(n-$ 2)-cycle on $L$ defined by

$$
\left\{\begin{array}{l}
l_{i}\left|L_{i}=l\right| L_{i} \\
l_{i} \mid L_{j}=0 \quad(i \neq j)
\end{array}\right.
$$

Let $H_{0}$ be a multiplicative free abelian group of $\operatorname{rank} \mu$, i.e. $H_{0}=$ $\Pi_{i=1}^{\mu} H_{i}$, where $H_{i}=\left(t_{i}:\right)(i=1,2, \cdots, \mu)$. Define a homomorphism $\psi_{0}$ of $G_{L}$ into $H_{0}$ by

$$
g^{l_{\gamma_{0}}}=\prod_{i=1}^{\mu} t_{i}^{\operatorname{link}\left(g, l_{i}\right)}
$$

Then, as before, we have the $d$ th elementary ideal $E_{d}\left(G_{L}, \psi_{0}\right)$ in $J H_{0}$, that is a topological invariant of the position of $l$ on $L$ in $S^{n} . E_{d}\left(G_{L}, \psi_{0}\right)$ will be denoted by $E_{d}[l]$.

Let $\sigma$ be a homomorphism of $H_{0}$ onto $H=\left(t\right.$ : ) defined by $t_{2}^{\sigma}=$ $t$. Since $g^{\psi}=t^{1 \text { ink }(g, l)}$, we have $\sigma \psi_{0}=\psi$. Since $\sigma$ is an homomorphism of $H_{0}$ onto $H$, we have the following theorem.

THEOREM 4. We have

$$
\left\{\begin{array}{l}
\left(E_{d}[l]\right)^{\sigma}=E_{d}(l) \text { and } \\
\left(E_{d}[l]\right)^{\circ}=\left(E_{d}(l)\right)^{\circ}
\end{array}\right.
$$


Now assume that $p_{n-2}(L) \geqq 1$. We have a sequence of homomorphism

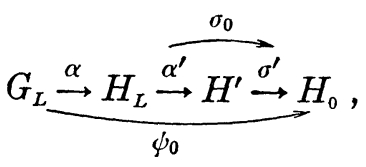

where $\alpha$ is the abelianizer of $G_{L}, H_{L} \cong H^{\prime}$ (a free abelian group of rank $\left.p_{n-2}(L)\right) \times\left(\right.$ the torsion subgroup of $H_{L}$ ) and $\alpha^{\prime}$ is the projection of $H_{L}$ onto $H^{\prime}, \psi_{0}=\sigma_{0} \alpha$, and $\sigma_{0}=\sigma^{\prime} \alpha^{\prime}$. Then, by Theorems 1 and $\mathrm{B}$, we have

$$
\begin{gathered}
E_{d}\left(G_{L}, \alpha^{\prime} \alpha\right) \subset E_{d}\left(H^{\prime}, i\right) \\
=\left\{\begin{array}{l}
(0), \text { if } d=0, \text { and } \\
\left(1-s_{1}, \cdots, 1-s_{p_{n-2}(L)}\right)^{p_{n-2}(L)-d}, \text { if } 1 \leqq d<p_{n-2}(L),
\end{array}\right.
\end{gathered}
$$

in $J H^{\prime}$, where we assume that $H^{\prime}$ is generated by $s_{1}, \cdots, s_{p_{n-2}(L)}$. In [2] it is proved that

$$
\left(1-s_{1}^{\sigma}, \cdots, 1-s_{p_{n-2}(L)}^{\sigma}\right) \subset\left(1-t_{1}, \cdots, 1-t_{\mu}\right) .
$$

Hence we have the following theorem.

Theorem 5. Assume that $p_{n-2}(L) \geqq 1$. Then we have

$$
\left\{\begin{array}{l}
E_{0}[l]=(0), \text { and } \\
E_{d}[l] \subset\left(1-t_{1}, \cdots, 1-t_{\mu}\right)^{p_{n-2}(L)-d}, \text { if } 1 \leqq d<p_{n-2}(L) .
\end{array}\right.
$$

THeorem 6. Assume that $p_{n-2}(L) \geqq 1$. Then we have

$$
\left\{\begin{array}{l}
E_{0}(l)=(0), \text { and } \\
E_{d}(l) \subset(1-t)^{p_{n-2}(L)-d}, \text { if } 1 \leqq d<p_{n-2}(L) .
\end{array}\right.
$$

REMARK. The first formula in Theorem 2 follows from Theorem 6 .

\section{On polyhedra in $S^{3}$.}

THEOREM $7^{1}$. Let $M^{3}$ be a 3-dimensional manifold and $L$ a polyhedron in $M^{3}$ that does not separate $M^{3}$. Then $\pi\left(M^{3}-L\right)$ has a presentation with deficiency $p_{1}\left(M^{3}-L\right)-p_{2}\left(M^{3}-L\right)$.

Proof. Let $K$ be a connected 2-dimensional polyhedron. Then $\pi(K)$ has a presentation with $\alpha_{1}-\left(\alpha_{0}-1\right)$ generators and $\alpha_{2}$ relators, where $\alpha_{i}(i=0,1,2)$ is the number of $i$-dimensional simplexes. Since $M^{3}-L$ has a connected 2-dimensional polyhedron as its deformation retract, say $K$, there is a presentation of $\pi\left(M^{3}-L\right)$ with deficiency 


$$
\begin{aligned}
\alpha_{1} & -\left(\alpha_{0}-1\right)-\alpha_{2}=1-p_{0}(K)+p_{1}(K)-p_{2}(K) \\
& =p_{1}\left(M^{3}-L\right)-p_{2}\left(M^{3}-L\right) .
\end{aligned}
$$

COROLLARY. Let $L$ be a polyhedron in $S^{3}$ that does not separate $S^{3}$. Then $\pi\left(S^{3}-L\right)$ has a presentation with deficiency 1 $p_{0}(L)+p_{1}(L)$.

Proof. By the Alexander duality theorem we have

$$
\begin{aligned}
p_{1}\left(S^{3}-L\right) & -p_{2}\left(S^{3}-L\right)=p_{1}(L)-\left(p_{0}(L)-1\right) \\
& =1-p_{0}(L)+p_{1}(L)
\end{aligned}
$$

THEOREM 8. Let $L$ be a polyhedron in $S^{3}$ that does not separate $S^{3}$. Then for each 1-cycle $l$ on $L$ we have

$$
E_{d}(l)=(0) \text {, if } d<1-p_{0}(L)+p_{1}(L) \text {. }
$$

REMARK. A greater number than $1-p_{0}(L)+p_{1}(L)$ can be obtained, if one (or more) component of $L$ is contractible in itself.

The following Theorem 9 and Theorem 10 are corollaries of Theorem 2 and Theorems 5 and 6 , respectively.

TheOREM 9. Let $L$ be a polyhedron in 3-sphere $S^{3}$ that does not separate $S^{3}$. Then for each 1-cycle $l$ on $L$ we have

$$
\left\{\begin{array}{l}
\left(E_{d}(l)\right)^{\circ}=(0), \text { if } d<p_{1}(L), \text { and } \\
\left(E_{d}(l)\right)^{\circ}=(1), \text { if } d \geqq p_{1}(L) .
\end{array}\right.
$$

THEOREM 10. Let $L$ be a polyhedron in 3-sphere $S^{3}$ that does not separate $S^{3}$. Assume that $p_{1}(L)>0$ and let $\mu=p_{0}(L)$. Then we have

$$
\left\{\begin{array}{l}
E_{0}[l]=0, \text { and } \\
E_{d}[l] \subset\left(1-t_{1}, \cdots, t-t_{\mu}\right)^{p_{1}(L)-d}, \text { if } 1 \leqq d<p_{1}(L)
\end{array}\right.
$$

Especially we have

$$
\left\{\begin{array}{l}
E_{0}(l)=0, \text { and } \\
E_{d}(l) \subset(1-t)^{p_{1}(L)-d}, \text { if } 1 \leqq d<p_{1}(L) .
\end{array}\right.
$$

4. Examples. Let $L_{1}$ be a $\theta$-curve, which is trivially imbedded in $S^{3}$. Then the fundamental group $G_{L_{1}}$ of $S^{3}-L_{1}$ is a free group of rank 2. From this it follows that for each 1-cycle $l$ on $L_{1}$ we have

$$
\left\{\begin{array}{l}
E_{d}(l)=(0), \text { if } d<2, \\
E_{d}(l)=(1), \text { if } d \geqq 2 .
\end{array}\right.
$$


Now let $L_{2}$ be a $\theta$-curve in $S^{3}$, which is shown in Fig. 1.

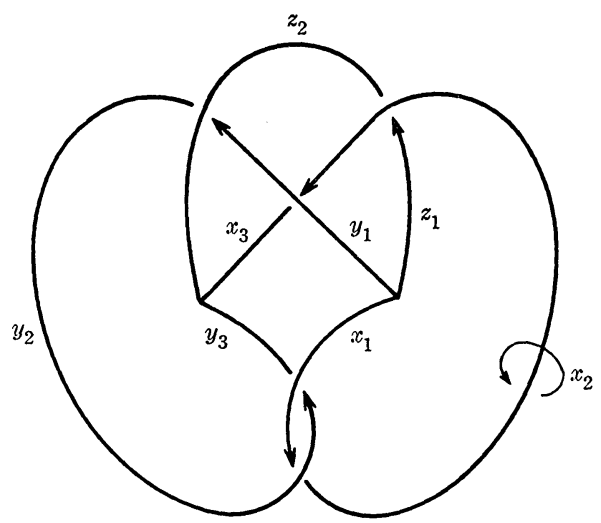

FIGURE 1

Then a presentation of the fundamental group $G_{L_{2}}$ of $S^{3}-L_{2}$ is as follows:

$$
\left(x_{1}, y_{1}, y_{2}: y_{2} x_{1} y_{1} y_{2}^{-1} x_{1} y_{2} y_{1}^{-1} y_{2}^{-1} x_{1}^{-1} y_{2} y_{1}^{-1} x_{1}^{-1} y_{2}^{-1} x_{1} y_{2} x_{1}^{-1}=1\right) \text {. }
$$

Now let $l$ be a 1-cycle on $L_{2}$ and suppose that link $\left(x_{1}, l\right)=c_{1}$ and link $\left(y_{1}, l\right)=\operatorname{link}\left(y_{2}, l\right)=c_{2}$. Then we have

$$
A\left(G_{L_{2}}, \psi\right) \approx\left(t^{c_{1}+c_{2}}+t^{c_{2}}+1,2,0\right) .
$$

Hence, we have

$$
\left\{\begin{array}{l}
E_{d}(l)=(0), \text { if } d<2, \\
E_{2}(l)=\left(t^{c_{1}+c_{2}}+t^{c_{2}}+1,2\right), \\
E_{d}(l)=(1), \text { if } d>2 .
\end{array}\right.
$$

This means that the position of $L_{1}$ and that of $L_{2}$ in $S^{3}$ are topologically inequivalent. Note that any one of three simple closed curves on $L_{2}$ is a trivial knot in $S^{3}$. (The example $L_{2}$ was also discussed in [4], but there was a mistake in calculation, that was pointed out by $R . H$. Fox to the author of the paper. Of course the underlying theory in that paper is also different to that of this paper as noted in the introduction.) We may also note that generally $E_{2}(l)$ of $l$ on $L_{2}$ in $S^{3}$ is not a principal ideal, that generally $E_{2}(l)$ does not satisfy the symmetricity property, that appears for knots and links in $S^{3}$. There are several of this kind of example in [6], too.

In another paper the author of the paper will prove that for any integral polynomial $f(t)$ with $f(t)= \pm 1$ there exists a $\theta$-curve $L$ in $S^{3}$ and a 1-cycle $l$ on $L$ such that $E_{2}(l)=(f(t))$.

5. On knots and links. Let $K$ be an oriented polyhedral ( $n-$ 
$2)$-sphere in $S^{n}(n \geqq 3)$ and $k$ an $(n-2)$-cycle on $K$ such that $k=c k^{\prime}$, where $k^{\prime}$ is the fundamental cycle of $K$. Consider the fundamental group $G_{K}$ of $S^{n}-K$ and its abelianization

$$
\alpha: G_{K} \rightarrow H_{K}=\left(t^{\prime}: \quad\right) .
$$

We choose the generator $t^{\prime}$ in such a way that for each $g \in G_{K}$

$$
g^{\alpha}=\left(t^{\prime}\right)^{\operatorname{link}\left(g, k^{\prime}\right)} .
$$

On the other hand, we have

$$
g^{\psi}=t^{1 \operatorname{ink}(g, k)}=t^{c\left(1 \mathrm{ink}\left(g, k^{\prime}\right)\right)} .
$$

Now define a homomorphism $\sigma$ of $\mathrm{H}_{K}$ into $H$ by $\left(t^{\prime}\right)^{\sigma}=t^{c}$. Then we have $\psi=\sigma \alpha$. From this it follows that

$$
E_{d}(k)(t)=E_{d}\left(k^{\prime}\right)\left(t^{c}\right)
$$

for each $d \geqq 0$. Further, since $H_{K}$ is infinite cyclic, we have $E_{0}(k)=$ (0) and $\left(E_{d}(k)\right)^{\circ}=(1)$ for $d \geqq 1$. If $n=3$, we have

$$
E_{1}(k)(t)=\left(\Delta_{K}\left(t^{c}\right)\right),
$$

where $\Delta_{K}(t)$ is the Alexander polynomial of the oriented simple closed curve $K$ in $S^{3}$.

Let $L$ be an oriented polyhedral $(n-2)$-link with $\mu$ components in $S^{n}(n \geqq 3)$, i.e. an ordered collection of $\mu$ number of mutually disjoint oriented polyhedral $(n-2)$-spheres in $S^{n}$. We assume that $\mu \geqq 2$. Let $L_{i}$ be the $i$ th component of $L$ for each $i(i=1, \cdots, \mu)$. Let $l$ be an $(n-2)$-cycle on $L$ and let $l_{i}^{\prime}$ be as follows:

$$
\left\{\begin{array}{l}
l_{i}^{\prime} \mid L_{i}=\text { the fundamental cycle on } L_{i}, \text { and } \\
l_{i}^{\prime} \mid L_{j}=0 \text {, if } i \neq j
\end{array}\right.
$$

Hence, we have $l=\sum_{i=1}^{\mu} c_{i} l_{i}^{\prime}$. Let $l_{i}=c_{i} l_{i}^{\prime}$ and $l^{\prime}=\sum_{i=1}^{\mu} l_{i}^{\prime}$. Consider the fundamental group $G_{L}$ of $S^{n}-L$ and its abelianization

$$
\alpha: G_{L} \rightarrow H_{L},
$$

which is a free abelian group of rank $\mu$. We choose the generator $t_{i}^{\prime}(i=1, \cdots, \mu)$ in such a way that for each $g \in G_{L}$

$$
g^{\alpha}=\prod_{i=1}^{\mu}\left(t_{i}^{\prime}\right)^{1 \mathrm{ink}\left(g, l^{\prime} i\right)} .
$$

On the other hand, we have

$$
g^{\psi_{0}}=\prod_{i=1}^{\mu} t_{i}^{\operatorname{link}\left(g, l_{i}\right)}=\prod_{i=1}^{\prime \prime}\left(t_{i}\right)^{\left.c_{i} \operatorname{link}(g, l)_{i}^{\prime}\right)} .
$$

Define a homomorphism $\sigma$ of $H_{L}$ into $H_{0}$ by $\left(t_{i}^{\prime}\right)^{\sigma}=t_{i}^{c} i$ for each $i(i=1$, $\cdots, \mu)$. Then we have $\psi_{0}=\sigma \alpha$. From this it follows that 


$$
E_{d}[l]\left(t_{1}, \cdots, t_{\mu}\right)=E_{d}\left[l^{\prime}\right]\left(t_{1}^{c_{1}}, \cdots, t_{\mu^{\mu}}^{c^{\mu}}\right) .
$$

Further we have $E_{0}(l)=0,\left(E_{d}(l)\right)^{\circ}=(0)$ for $d<\mu$, and $\left(E_{d}(l)\right)^{\circ}=(1)$ for $d \geqq \mu$. If $n=3$, we have

$$
E_{1}[l]\left(t_{1}, \cdots, t_{\mu}\right)=\left(1-t_{1}^{c_{1}}, \cdots, 1-t_{\mu^{\mu}}^{c^{\mu}}\right) \Delta_{L}\left(t_{1}^{c_{1}}, \cdots, t_{\mu^{\mu}}^{c^{\mu}}\right),
$$

where $\Delta_{L}\left(t_{1}, \cdots, t_{\mu}\right)$ is the Alexander polynomial of the link $L$ in $S^{3}$ and, hence

$$
E_{1}(l)(t)=\left(1-t^{c}\right) \Delta_{L}\left(t^{c_{1}}, \cdots, t^{c_{\mu}}\right),
$$

where $c=$ g.c.d. $\left(c_{1}, \cdots, c_{\mu}\right)$.

REmark. Further, it is proved by Shinohara and Sumners [5] that if $n \geqq 4, E_{d}(l)=(0)$ for $d<\mu$.

6. On a Torres' formula for a link. The following consideration may be interesting: Suppose that $L$ is an oriented link with multiplicity $\mu(>1)$ in $S^{3}$ and $L_{i}$ the $i$ th component of $L .(i=1,2$, $\cdots, \mu)$. Let $l$ be a 1 -cycle on $L$ and express $l$ as $\sum_{i=1}^{\prime \prime} l_{i}$ as before. Denote $\sum_{i=1}^{\mu-1} l_{i}$ by $l^{*}$. Now let $L^{*}=L-L_{\mu}$ and let $l^{*}$ be the 1-cycle on $L^{\sharp}$ such that $l^{*}=l \mid L^{\sharp}$. Hence we have $l^{*}=l^{*} \mid L^{\sharp}$. Let $H_{0}$ be a multiplicative free abelian group of rank $\mu$, i.e. $H_{0}=\prod_{i=0}^{\mu} H_{i}$, where $H_{i}=\left(t_{i}:\right)(i=1,2, \cdots, \mu)$. Let $H_{0}^{*}=\prod_{i=1}^{\mu-1} H_{i}$.

Now let $\psi_{0}$ be the homomorphism of $G_{L}$ into $H_{0}$ such that for each $g \in G_{L}$

$$
g^{\not<0}=\prod_{i=1}^{\mu} t_{i}^{\operatorname{link}\left(g, l_{i}\right)},
$$

$\psi_{0}^{*}$ the homomorphism of $G_{L}$ into $H_{0}$ such that for each $g \in G_{L}$

$$
g^{\psi \psi_{0}^{*}}=\prod_{i=1}^{\mu-1} t_{i}^{1 \operatorname{link}\left(g, l_{i}\right)}
$$

and $\psi_{0}^{\sharp}$ the homomorphism of $G_{L}^{\sharp}$ into $H_{0}^{\sharp}$ such that for each $g \in G_{L}^{\ddagger}$

$$
g^{\psi_{0}^{*}}=\prod_{i=1}^{\mu-1} t^{\operatorname{link}\left(g, l_{i}\right)} .
$$

Let $c_{i}$ be the coefficient of $l$ on $L_{i}(i=1, \cdots, \mu)$. Then we have the following theorem.

Theorem 11. We have

$$
E_{1}\left[l^{*}, \psi_{0}^{*}\right]=\left(t_{1}^{c_{1} l_{1 \mu}} \cdots t_{t^{\prime}-1}^{c \mu-1} l_{\mu-1 / \mu}-1\right) E_{1}\left[l, \psi_{0}^{\sharp}\right],
$$

where $l_{i j}=\operatorname{link}\left(L_{i}, L_{j}\right)(i, j=1,2, \cdots, \mu)$. 


$$
E_{1}\left[l, \psi_{0}\right]=\left(t_{1}^{c_{1}}-1, \cdots, t_{\mu^{\mu}}^{c_{\mu}}-1\right) \Delta_{L}\left(t_{1}^{c_{1}}, \cdots, t_{\mu^{\mu}}^{c^{\mu}}\right) .
$$

Then we have

$$
E_{1}\left[l^{*}, \psi_{0}^{*}\right]=\left(t_{1}^{c_{1}}-1, \cdots, t_{\mu-1}^{c_{\mu-1}}-1\right) \Delta_{L}\left(t_{1}^{c_{1}}, \cdots, t_{\mu-1}^{c_{\mu-1}}, 1\right) .
$$

On the other hand, we have

$$
E_{1}\left[l^{\sharp}, \psi^{\sharp}\right]=\left(t_{1}^{c_{1}}-1, \cdots, t_{\mu-1}^{c_{\mu-1}}-1\right) \Delta_{L^{\sharp}}\left(t_{1}^{c_{1}} \cdots, t_{\mu-1}^{c \mu-1}\right) .
$$

A Torres' formula of the Alexander polynomial of links ([7]) is as follows:

If $\mu=2$, then

$$
\Delta_{L}\left(t_{1}, 1\right)=\Delta_{L^{\sharp}}\left(t_{1}\right)\left(t_{1}^{l_{12}}-1\right) /\left(t_{1}-1\right) .
$$

If $\mu>2$, then

$$
\Delta_{L}\left(t_{1}, \cdots, t_{\mu-1}, 1\right)=\left(t_{1}^{l_{1 \mu}} \cdots t_{\mu-1}^{l_{\mu-1 \mu}}-1\right) \Delta_{L^{\sharp}}\left(t_{1}, \cdots, t_{\mu-1}\right) .
$$

Hence if $\mu>2$, the statement of the theorem follows immediately. If $\mu=2$ and $c_{1} \neq 0$, we have

$$
\begin{aligned}
& E_{1}\left[l^{*}, \psi_{0}^{*}\right]=\left(t_{1}^{c_{1}}-1\right) \Delta_{L}\left(t_{1}^{c_{1}}, 1\right) \\
= & \left(t_{1}^{c_{1} l_{12}}-1\right) \Delta_{L^{\sharp}}\left(t_{1}^{c_{1}}\right)=\left(t_{1}^{c_{1} l_{12}}-1\right) E_{1}\left[l^{\sharp}, \psi_{0}^{\sharp}\right] .
\end{aligned}
$$

The statement of the theorem is trivial, if $c_{1}=0$ and $\mu=2$. Thus the proof of the theorem is complete.

REMARK. Theorem 11 can be proved directly and the Torres' theorem can be obtained as a corollary to this theorem.

\section{REFERENCES}

1. R. H. Crowell and R. H. Fox, Introduction to Knot Theory, Ginn and Company, 1963.

2. R. H. Fox, Free differential calculus, I, Ann. of Math., 57 (1953), 547-560.

3. - Free differential calculus, II, Ann. of Math., 59 (1954), 145-518.

4. S. Kinoshita, Alexander polynomials as isotopy invariants, I, Osaka Math. J., 10 (1958), 263-271.

5. Y. Shinohara and D. W. Sumners, Homology invariants of cyclic coverings with application to links (to appear in the Trans. Amer. Math. Soc.).

6. S. Suzuki, On linear graphs in 3-sphere, Osaka J. Math., 7 (1970), 375-396.

7. G. Torres, On the Alexander polynomial, Ann. of Math., 57 (1954), 145-158.

Received May 4, 1970 and in revised form January 13, 1972. The author of this paper was partially supported by NSF Grants GP-11943 and GP-19964. 


\section{PACIFIC JOURNAL OF MATHEMATICS}

\section{EDITORS}

H. SAMELSON

Stanford University

Stanford, California 94305

C. R. HobBY

University of Washington

Seattle, Washington 98105
J. DUGUNDJI

Department of Mathematics

University of Southern California

Los Angeles, California 90007

RICHARD ARENS

University of California

Los Angeles, California 90024

\section{ASSOCIATE EDITORS}

E. F. BECKENBACH

B. H. NeumanN

F WoLF

K. YoshidA

\section{SUPPORTING INSTITUTIONS}

UNIVERSITY OF BRITISH COLUMBIA

UNIVERSITY OF SOUTHERN CALIFORNIA

CALIFORNIA INSTITUTE OF TECHNOLOGY

UNIVERSITY OF CALIFORNIA

MONTANA STATE UNIVERSITY

STANFORD UNIVERSITY

UNIVERSITY OF NEVADA

NEW MEXICO STATE UNIVERSITY

OREGON STATE UNIVERSITY

UNIVERSITY OF OREGON

OSAKA UNIVERSITY

UNIVERSITY OF TOKYO

UNIVERSITY OF UTAH

WASHINGTON STATE UNIVERSITY

UNIVERSITY OF WASHINGTON

$\stackrel{*}{*} \stackrel{*}{*} \stackrel{*}{*}{ }^{*}{ }^{*}$ MMEICAN MATHEMATICAL SOCIETY

NAVAL WEAPONS CENTER

Printed in Japan by International Academic Printing Co., Ltd., Tokyo, Japan 


\section{Pacific Journal of Mathematics}

\section{Vol. 42, No. $1 \quad$ January, 1972}

Tage Bai Andersen, On Banach space valued extensions from split faces ........

David Marion Arnold, A duality for quotient divisible abelian groups of finite

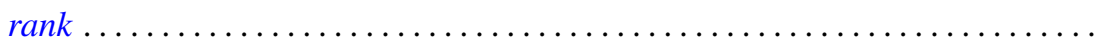

Donald Pollard Ballou, Shock sets for first order nonlinear hyperbolic

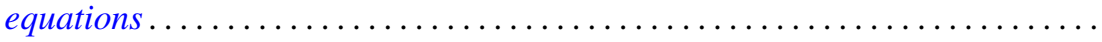

Leon Brown and Lowell J. Hansen, On the range sets of $H^{p}$ functions .........

Alexander Munro Davie and Arne Stray, Interpolation sets for analytic

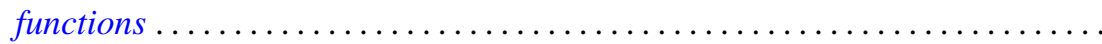

M. G. Deshpande, Structure of right subdirectly irreducible rings. II . . . . . . . . .

Barry J. Gardner, Some closure properties for torsion classes of abelian

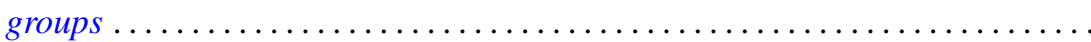

Paul Daniel Hill, Primary groups whose subgroups of smaller cardinality are

direct sums of cyclic groups . . . . . . . . . . . . . . . . . . .

Richard Allan Holzsager, When certain natural maps are equivalences .........

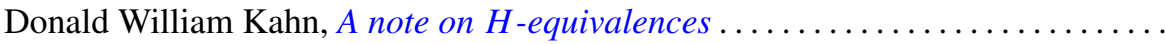

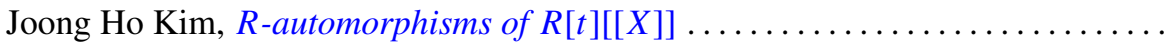

Shin'ichi Kinoshita, On elementary ideals of polyhedra in the 3-sphere.........

Andrew T. Kitchen, Watts cohomology and separability...

Vadim Komkov, A technique for the detection of oscillation of second order

ordinary differential equations .

Charles Philip Lanski and Susan Montgomery, Lie structure of prime rings of characteristic 2

Andrew Lenard, Some remarks on large Toeplitz determinants . .

Kathleen B. Levitz, A characterization of general Z.P.I.-rings. II .

Donald A. Lutz, On the reduction of rank of linear differential systems

David G. Mead, Determinantal ideals, identities, and the Wronskian ...

Arunava Mukherjea, A remark on Tonelli's theorem on integration in product

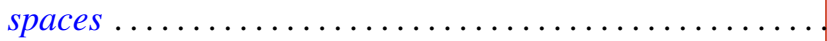

Hyo Chul Myung, A generalization of the prime radical in nonassociative rings.

John Piepenbrink, Rellich densities and an application to unconditionally nonoscillatory elliptic equations.

Michael J. Powers, Lefschetz fixed point theorems for a new class of multi-valued maps .

Aribindi Satyanarayan Rao, On the absolute matrix summability of a Fourier

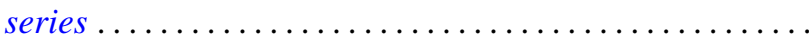

T. S. Ravisankar, On Malcev algebras ......................... 227

William Henry Ruckle, Topologies on sequences spaces . . . . . . . . . . . . . 235

Robert C. Shock, Polynomial rings over finite dimensional rings . . . . . . . . . 251

Richard Tangeman, Strong heredity in radical classes . . . . . . . . . . . . . . 259

B. R. Wenner, Finite-dimensional properties of infinite-dimensional spaces . . . . 267 